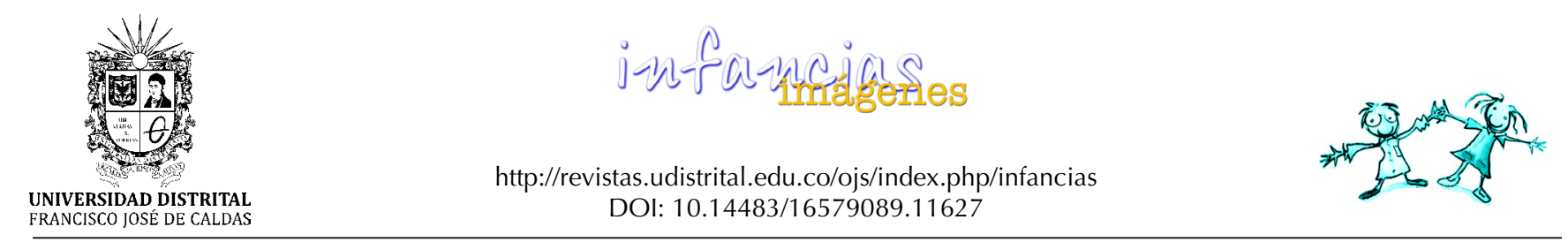

IMÁGENES DE INVESTIGACIÓN

\title{
La seguridad escolar como problema político*
}

\author{
School Safety as a Political Problem \\ Daniel Enrique Carranza Torres ${ }^{1}$
}

Para citar este artículo: Carranza, D. E. (2017). La seguri-

Recibido: 15-febrero-2015 / Aprobado: 24-abril-2017

dad escolar como problema político. Infancias Imágenes,

16(1), 9-24.

\section{Resumen}

La seguridad escolar comienza a cobrar relevancia en cuanto problema político a finales de la década de 1980 en Europa, el discurso jurídico internacional impulsa y va definiendo la intervención de la política de seguridad escolar en Latinoamérica a inicios del siglo XXI. En este artículo se presentan las miradas públicas sobre el tema en algunos países, en particular Colombia y México. En el debate sobre la seguridad escolar es necesario tener presente la influencia que ejerce la doctrina de seguridad nacional en el mundo, su posterior desplazamiento a lo urbano o local, lo cual se equipara a la seguridad ciudadana que, influenciada a su vez por el enfoque de derechos humanos, permitirá entender las diferencias entre el paradigma de la seguridad humana y la doctrina de la seguridad nacional; así, se evidencian los desafíos de la seguridad escolar en el campo educativo. El estado del arte permitirá identificar la relevancia de la seguridad escolar para la ciudad de Bogotá, como respuesta integral a las problemáticas de violencia escolar y su configuración como problema político.

Palabras clave: seguridad humana; desarrollo social; derechos humanos; ciudadanía; convivencia pacífica; política pública; problemas sociales.

\begin{abstract}
Albstract
School security began to become relevant as a political problem in the late 1980s in Europe; the international legal discussion is prompted and defined the intervention of school security policy in Latin America at the beginning of the 21 st century. This article presents public views on the subject in some countries, particularly Colombia and Mexico. In the debate over school safety it is necessary to bear in mind the influence exercised by the doctrine of national security in the world, its subsequent displacement to urban or local level, which equates to citizen security-influenced in turn by the human rights approach—which will allow the understanding differences between human security paradigm and national security doctrine. Thus, challenges of school safety in educational realm are evidenced. State of the art will allow identification relevance of school security for Bogotá, as an integral response to the problems of school violence and its configuration as a political problem.
\end{abstract}

Keywords: human security; social development; human rights; citizenship; peaceful coexistence; government policy; social problems.

\footnotetext{
* Este artículo de revisión corresponde al trabajo de investigación de tesis doctoral del autor como parte del estado del arte de su proyecto que se encuentra en curso. "Política de seguridad escolar en Bogotá: una década de implementación (2005-2015)". Doctorado Interinstitucional en Educación. Universidad Distrital Francisco José de Caldas.

1 Trabajador Social. Magíster en Investigación Social Interdisciplinaria. Doctorando en Educación Universidad Distrital Francisco José de Caldas. Catedrático, Maestría en Educación, Universidad Libre de Colombia. Correo electrónico: tscarranza@gmail.com
} 


\section{Introducción}

Tomar como centro de análisis la perspectiva de la seguridad escolar implica entender su lógica de construcción como un problema relevante de carácter político, en el que se pueden establecer cercanías con los conceptos de seguridad nacional, seguridad ciudadana y, de forma más significativa, su vínculo con la concepción de la seguridad humana.

La configuración de la seguridad escolar como un problema político pasa por el entendimiento, entre otros, de la violencia escolar y los derechos humanos, fundamentalmente estos dos aspectos vistos desde la óptica del interés superior de la infancia; en este sentido, el discurso de la seguridad escolar cobra fuerza por su estrecha relación con el enfoque de derechos de los niños, que se consagra en la Asamblea General de las Naciones Unidas, que adoptó la Convención sobre los Derechos de los Niños, el 20 de noviembre de 1989.

A partir de esta Convención, los gobiernos desarollan sus políticas para la niñez y la adolescencia. El Fondo de las Naciones Unidas para la Infancia (Unicef) (2005) plantea:

[...] el marco conceptual que proporciona la Convención permite un análisis más profundo de las causas subyacentes que dan lugar a las altas tasas de mortalidad y morbilidad en la infancia y en la niñez, a la marginación de grupos completos de niños, el abuso infantil y la violencia contra los niños, niñas y adolescentes, entendidos ahora como la manifestación de violaciones generalizadas a los derechos del niño. (p. 3).

En el campo escolar la violación a los derechos de los niños y las niñas se configura de diferentes formas; de esto, el discurso jurídico da cuenta, en parte, a partir de entender la nueva condición de sujetos de derecho que gozan los niños y niñas, sin embargo el alcance o efectividad de las políticas de cada Estado frente a esta responsabilidad está supeditado al desarrollo de su doctrina legislativa, en primera instancia, luego a los factores de contexto de cada país, esto incluye las capacidades económicas, sociales y culturales del mismo, que se ven necesariamente reflejadas en los procesos democráticos y participativos para el diseño y ejecución de estas políticas garantistas.

Dicho así, se hace necesario una revisión permanente de las políticas públicas que abordan este tema en el mundo, en Colombia y, particularmente, en una ciudad como Bogotá que ha implementado durante los últimos diez años políticas de seguridad escolar. Identificar la necesidad de ajustar o reformular la política frente a su eficacia en la aplicación de los derechos de niños y niñas implica superar la visión coercitiva que puede llegar a imponer una mirada centrada en la lógica de la seguridad nacional.

\section{De la seguridad nacional a la seguridad escolar}

El concepto de seguridad tiene un desarrollo en la lógica de construcción del Estado-nación, lo que hace indispensable remitirse a la seguridad nacional como categoría política referida al Estado; esta, como tal, se consolida durante la segunda mitad del siglo XX y sirve de soporte a la tendencia militarista de la segunda posguerra mundial. Equipara la seguridad del Estado con la de la sociedad, pero las transformaciones que desde la década de 1960 vienen sucediendo, en lo económico, político y tecnológico, amplían las dimensiones estratégicas internacionales, en las cuales la categoría toma sentido (Leal, 1994, p. 45).

Estas transformaciones implican un tratamiento multi e interdisciplinario a los problemas de seguridad frente a los componentes básicos de la globalización; es decir, el final de la Guerra Fría, la multipolaridad económica y política, junto con la unipolaridad militar estadounidense, el resurgimiento de los nacionalismos y la emergencia de los conflictos regionales y locales, el debilitamiento del Estado, base del paradigma dominante de organización política (Estado-nación).

La doctrina de la seguridad nacional ha tenido un impacto significativo en el diseño de las políticas gubernamentales de seguridad en Colombia a partir de la segunda posguerra mundial. Esta doctrina ha estado, desde su concepción, íntimamente ligada a la política exterior norteamericana. (Borda, Rodríguez y Libreros, 2004, p. 40). 
La idea de la seguridad nacional fue ampliamente difundida e impulsada por los Estados Unidos de América en el marco de la Guerra Fría. Incluso, Ilegó a incorporarse en tratados internacionales en el ámbito americano como el Tratado Interamericano de Asistencia Recíproca (TIAR) (Angarita, 2002, p. 126). Estas nuevas circunstancias redefinen la labor castrense, la cual se concentra más en asuntos políticos internos, entrabando así el desarrollo democrático.

La militarización de la política consiste, precisamente, en calificar y tratar como militares los problemas políticos y sociales más variados, sustituyendo su solución por medidas generalmente represivas, que agravan con el tiempo esos problemas y tergiversan el ideal democrático. (Leal, 1994, p. 45).

Esta tergiversación entre las formas de abordar los conflictos de carácter social con los instrumentos legítimos de coerción del Estado, más precisamente sus fuerzas armadas y de policía, ha generado una confusión entre la seguridad del Estado, equiparándola con la de sus ciudadanos; este equívoco es manifiesto en la segunda estrategia de seguridad, emanada en mayo de 1993, en el gobierno de César Gaviria, teniendo en cuenta que su primer capítulo reveladoramente se titula "La seguridad del Estado es también la seguridad de la gente" (Camacho, 1994, p. 85).

La cuestión es que más allá de la seguridad del Estado y de su gobierno, que se fundamenta en cuanto las amenazas externas en su ejército, está la seguridad como tarea política; aquí la seguridad de los ciudadanos del régimen se va construyendo, no con el fortalecimiento del brazo armado de la república, sino con el conjunto de las políticas estatales, particularmente las Ilamadas sociales. En esencia, se puede pensar en la aplicación de la biopolítica, mediante la instauración de mecanismos reguladores de la vida social,

[...] para asegurar concretamente el principio de seguridad es necesario recurrir a una serie de técnicas de vigilancia de los individuos, un diagnóstico de lo que estos son, clasificación de su estructura mental y de su patología. De allí, la inicial aversión hacia los pobres, pues la anormalidad deviene inicialmente de la pobreza. (Jiménez, 2013, p. 19).

Lo anterior es un giro al abordaje de la seguridad como dispositivo de control social, pero en un tránsito lento, es decir, asociado a la aparición de políticas sociales. Por ello,

[...] el aspecto central para todas las posibles nuevas líneas conceptuales de la seguridad parte de la necesidad de los individuos de vivir en paz y de contar con los medios económicos, políticos y ambientales para una existencia digna, en un contexto en el cual la seguridad interna sea un asunto político y no militar. (Leal, 1994).

Sin embargo,

[...] la seguridad interna dio prioridad a la seguridad del Estado y edificó para ello un andamio institucional pensado para el control político y social a través del ejercicio de la fuerza, con lo cual las funciones de orden público dieron preferencia al enfoque represivo, en desmedro de la protección del individuo. (Borda, Rodríguez y Libreros, 2004, p. 9).

Llegados los años 1990, la doctrina de seguridad nacional perdió vigencia debido a los cambios en el orden mundial, el fin de la bipolaridad económica y militar fue trascendental en la pertinencia de la categoría y su desplazamiento o equiparación hacia la categoría de seguridad ciudadana, dirigida a problemas de orden público en ciudades o centros urbanos.

Los cambios en Colombia frente a la doctrina de seguridad nacional, terminada la década de 1990 no se hicieron esperar; la Corte Constitucional en su Sentencia C-251 del 11 de abril de 2002, declaró la inconstitucionalidad de la Ley 684 de 2001, por la cual se creó el sistema de seguridad y defensa nacional. "Dicha Ley fue declarada contraria a la Constitución por la Corte Constitucional, con el argumento central que significaba la instauración de la doctrina de la seguridad nacional vedada por la Carta Política de 1991" (Borda, Rodríguez y Libreros, 2004, p. 9). 
Así, para abordar el concepto de seguridad ciudadana es indispensable remitirse a la seguridad nacional; por la emergencia de los conflictos regionales y locales, el problema de la seguridad adquiere la comprensión de los componentes básicos de la globalización; en este sentido, estamos frente a la manifestación tangible de una sociedad de control,

[...] aquella que se sitúa en el borde último de la modernidad, en la cual los mecanismos de dominio se vuelven más democráticos y más inmanentes al campo social, y se distribuyen completamente por la mente y el cuerpo de los individuos. La sociedad de control da cuenta de la declinación de la soberanía de los Estados nacionales modernos. (Jiménez, 2013, p. 22).

Después del 11 de septiembre de 2001, por cuenta de los atentados a las Torres Gemelas en Estados Unidos, se reencaucha la seguridad nacional y se equipara a la lucha contra el errorismo, y la se12 guridad ciudadana, sencillamente termina siendo la expresión de esta en el contexto local, protección al mercado y recuperación, captación de escenarios productivos.

En cuanto al contexto hemisférico en América Latina, los Estados Unidos, siguiendo los lineamientos de su Estrategia de Seguridad Nacional de septiembre de 2002, sobre la guerra preventiva contra sus enemigos directos, ha orientado su política a identificar fuentes de terrorismo en el hemisferio y a incrementar su presión sobre algunos gobiernos en el área para que combatan más eficazmente este fenómeno en conjunto con su estrategia de lucha contra el narcotráfico.

En el ámbito regional, la estrategia estadounidense, promovida también por algunos gobiernos, como el de Colombia, donde se encuentra el mayor foco de violencia y desestabilización de la región, así como de movimiento del narcotráfico, consiste en vincular al narcotráfico como fuente de financiamiento de los grupos terroristas locales y unificar políticas y recursos para enfrentar ambos fenómenos.

Este proceso, donde la seguridad es el elemento fundamental de las relaciones de Estados Unidos con la región, genera distanciamientos entre los gobiernos cercanos a Washington y aquellos que tienen una línea más independentista, como Colombia y Venezuela, ambos en los extremos.

La Cumbre de Jefes de Estado del hemisferio, celebrada en Monterrey en octubre de 2003, significó un termómetro de las relaciones entre Estados Unidos y América Latina, las cuales indican que esta última región fue la gran perdedora del 11 de septiembre.

En efecto, con posterioridad a los atentados terroristas, América Latina dejó de estar en la agenda directa del gobierno de Washington, más preocupado por la lucha contra el terrorismo internacional y también en renovar su alianza con la Unión Europea, luego de la crisis que tuvieron a raíz de la invasión a Irak. Pero, a pesar de este repliegue a la concepción más conservadora de la seguridad en el mundo, es claro y pertinente que la seguridad ciudadana ha de ser conceptuada

[...] como un instrumento de bienestar social. Así concebida, sobrepasa el estricto marco jurídico-social del sistema penal tradicional; ya no son solamente la justicia y la policía quienes han de procurarla, sino también las políticas de bienestar social y calidad de vida junto con otros nuevos actores de control. (Delgado, 1997, p. 96).

Este enfoque es Ilamado sociopolítico, ya que

[...] la seguridad ciudadana, más allá del carácter de tema ideologizado, es ante todo un derecho al que le corresponde un deber. Junto con la defensa de las amenazas externas y las garantías de mantenimiento de la paz y el orden, la seguridad material constituye uno de los pilares sobre los cuales se asientan las relaciones entre gobernantes y gobernados. (Camacho, 1994, p. 70).

La seguridad ciudadana, concebida como seguridad humana que propicia la realización de los derechos y libertades fundamentales de todas las personas, es diferente de la seguridad del Estado. Para el Estado existe el deber, e incluso el derecho, de proteger su existencia e integridad de los ataques que se den por fuera de las reglas establecidas en el ordenamiento constitucional y en las normas 
pertinentes del derecho internacional, o que apelen a la violencia (Borda, Rodríguez y Libreros, 2004, p. 16).

En el mundo a mediados de la década de 1990 e inicios de los años 2000, toma fuerza la categoría de seguridad humana, es decir tiene un corto tiempo de aparición y se presenta en parte como antagónica a la seguridad nacional y mucho más amplia que la concepción de seguridad ciudadana, pues se concentra fundamentalmente en la seguridad de la persona y no del Estado o del mercado.

La seguridad humana se va configurando como un paradigma, según Pérez de Armiñon (2007), existen dos enfoques que deben ser punto de partida para un entendimiento de la categoría: el amplio y el restringido:

El primero se centra en la "libertad frente a la necesidad", esto es, en la satisfacción del desarroIlo humano y de un mínimo bienestar (seguridad alimentaria, sanitaria, medioambiental, etc.), y se corresponde a la formulación inicial de la seguridad humana realizada por el PNUD en su Informe sobre Desarrollo Humano de 1994, sostenida también por la Comisión de la Seguridad Humana, liderada por Japón, y su relevante informe "La seguridad humana ahora", publicado en 2003. Por su parte, el enfoque restringido se centra en la "libertad frente al temor", esto es, en la protección ante la violencia física en contextos de conflicto. Su gestación y expansión ha venido de la mano en gran medida de la Red de Seguridad Humana, promovida por Canadá e integrada por varios estados. (p. 60).

El enfoque de los derechos humanos y la apuesta por el desarrollo humano constituirán dos pilares en el entendimiento de la seguridad humana, después de 1994, aparecerán dos hitos que fortalecerán la categoría de seguridad humana,

[...] la creación, en 1999, de la Red de Seguridad Humana, formada por doce países de todas las regiones del mundo, que celebra reuniones ministeriales cada año; y la publicación, en 2003, del informe de la Comisión de Seguridad Humana titulado "Human Security Now: Protecting and Empowering People" (La seguridad humana ahora: proteger y habilitar a la gente), en el que se insta a adoptar una iniciativa mundial para promover la seguridad humana. (Fuentes y Rojas, 2005, p. 10).

La emergencia para el contexto latinoamericano frente a la categoría de seguridad humana se hace pertinente pero también ambigua, por la dificultad que supone llevar a la práctica desde la institucionalidad pública todo el despliegue que implica una categoría tan amplia. Por lo anterior la Organización de Estados Americanos (OEA) plantea:

[...] en nuestro hemisferio, en nuestra condición de Estados democráticos comprometidos con los principios de la carta de las Naciones Unidas y la Carta de la OEA, reafirmamos que el fundamento y razón de ser de la seguridad es la protección de la persona humana. La seguridad se fortalece cuando profundizamos su dimensión humana. Las condiciones de la seguridad humana mejoran mediante el pleno respeto a la dignidad, los derechos humanos y las libertades fundamentales de las personas, así como mediante la promoción del desarrollo económico y social, la inclusión social, la educación y la lucha contra la pobreza, las enfermedades y el hambre. (OEA, 2003, p. 3).

En este sentido se comparte el análisis que hacen Fuentes y Rojas (2005):

[...] en el contexto latinoamericano habría que concentrarse en dos temas: a) la necesidad de establecer en términos prácticos y operativos la relación entre la seguridad nacional, la seguridad internacional y la seguridad humana; b) el uso de la violencia como un elemento determinante de análisis en esta materia. (p. 15).

Hasta aquí existe una línea que relaciona a estos tipos de seguridad, que va de la concepción de seguridad nacional, pasando por el carácter híbrido de la seguridad ciudadana, por cuanto combina la coerción y cohesión social garantizada por el Estado, la emergencia del paradigma de la seguridad humana, en su búsqueda por garantizar los derechos humanos, y por último, la seguridad escolar, que amparada en los cambios que produjo la CDN 
(Convención sobre los Derechos del Niño, 1989) en el campo educativo se debate entre poner en acción el enfoque de derechos humanos, o responder a la eliminación de amenazas, y del control sanción, característico de la doctrina de seguridad nacional.

\section{La seguridad escolar como problema político}

El enlace clave, es decir, la línea que existe entre las concepciones de seguridad hasta aquí esbozadas y la seguridad escolar, tiene que ver con la manifestación de la violencia social y su relación con el campo educativo. Según Gvirtz y Larrondo (2012),

[...] la violencia y la escuela tienen una relación de muy larga data, si bien a lo largo del tiempo fue cambiando el tipo de violencia y su modo de ejercicio. Por ejemplo, a fines del siglo XIX y principios del siglo XX —o mediados de siglo— el ejercicio de la violencia estaba prácticamente monopolizado por la autoridad formal, ya que era prerrogativa exclusiva de los docentes o directivos. (p. 296).

A su vez, hay que tener presente que los contextos y entornos de la escuela son fundamentales en la comprensión de su seguridad escolar; así,

[...] según el primer planteamiento de Henry Giroux, las escuelas no pueden ser analizadas como instituciones separadas del contexto socioeconómico en que están situadas, es por ello que se hace difícil pensar la escuela en sí misma; por lo tanto, es necesario dar cuenta del entorno en el cual se desenvuelve, en un contexto que está en permanente cambio y con unos sujetos allí involucrados que, en su conjunto, afectan directamente las relaciones en el interior de la dinámica escolar, al tiempo que esta puede incidir en lo comunitario. (Ruiz y Amariles, 2006, p. 81).

Es pertinente mencionar que existen relaciones muy estrechas entre el entorno y la escuela, como en este caso el sentimiento de inseguridad y la violencia. Catherine Blaya (2012) identifica estos dos fenómenos:
[Primero] el sentimiento de inseguridad en el barrio genera un sentimiento de inseguridad en el colegio también y que los dos se encuentran significativamente relacionados. Además, el sentimiento de inseguridad está relacionado con la percepción de vivir en un lugar poco agradable. (p. 43).

[Segundo,] cuando la percepción de violencia en el barrio es alta, la tendencia es parecida en cuanto a lo que pasa en el colegio. (p. 42).

La categoría de seguridad escolar en la actualidad tiene más elementos que complejizan su comprensión, como ya se aclaró, recurriendo a la influencia perniciosa del entorno escolar, a la manifestación directa de la violencia social, al microtráfico de estupefacientes, al pandillismo, a la desigualdad, etc. Esto va mucho más allá de lo que se pensó en sus inicios, cuando dicha categoría comenzó a configurarse como relevante en el mundo. En los Países Bajos, por ejemplo, comenzó una campaña de escuelas seguras, con una fuerte inclinación a identificar a los jóvenes en riesgo frente a conductas antisociales; así, la idea de seguridad escolar en el mundo aparece por primera vez como un problema político.

El 22 de junio de 1995 este programa se publicó bajo el título Voorkoming en bestijding geweld in scholen (Prevención y lucha contra la violencia en la escuela). El Ministerio difundió al mismo tiempo un folleto sobre Escuelas Seguras (Ministerie van Onderwijs, Cultuur en Wetenschappen, 1995a, 1995b). La campaña nacional Escuelas Seguras (ver apartado Estrategias que inciden sobre los escolares y la escuela) fue iniciada por el Ministerio al mismo tiempo. En 1995 el Ministerio de Salud, Bienestar y Deporte publicó un informe referente a la extracción y características de los jóvenes pertenecientes al grupo de riesgo en relación al desarrollo de la conducta antisocial y las políticas al respecto (Schuyt, 1995). El informe hacía hincapié en la detección temprana (observación) y apoyo efectivo para esos muchachos. (Mooij, 1997, p. 44).

Así mismo,

[...] en la Conferencia de Utrecht los investigadores de los países asistentes decidieron formar la red 
de estudio Gestión y Evaluación de la Seguridad en la Escuela. El primer objetivo de la misma es cooperar en la investigación y desarrollo de escuelas seguras, intercambiar información pertinente y asistir a cada uno en el desarrollo y evaluación de proyectos internacionales. (p. 49).

Esta preocupación por la seguridad escolar fue configurándose en el caso francés, de forma más moderada hacia el asunto de la falta de civismo, separando el sentimiento de inseguridad y la victimización. No se trataba de cambiar una categoría por otra; Debarbieux (1997) lo plantea al reconocer que la falta de civismo genera un estado de inseguridad que puede ser un propiciador de hechos o actos de violencia escolar (p. 87).

La preocupación por la seguridad escolar traspasa rápidamente fronteras y llega a un momento crucial donde el Consejo de la Unión Europea se pronuncia al respecto. En 1997 este Consejo,

[...] hace un Ilamamiento a todos sus estados miembros afirmando que la seguridad en las escuelas afecta y es un factor determinante de la calidad de la educación, dado que no puede esperarse suficiente calidad en la educación sin un clima abierto y seguro que permita a los alumnos y profesores aprender y enseñar sin la preocupación de ser intimidados, tiranizados o maltratados. Además, el Consejo señala la importancia que tiene la participación de los padres, alumnos, profesores, directores e instituciones locales para el éxito de la escuela. (Unión Europea, 1997). (Monclús y Saban, 2006, p. 60).

Sin duda, en este momento se conforman investigaciones y experiencias que poco a poco configuran un campo de estudio importante para el sector educativo y la sociedad en general.

En los 90, por un lado, a consecuencia de las demandas del alumnado de secundaria sobre la necesidad de seguridad y, por otro, de la influencia de la literatura científica anglófona, se va tomando conciencia tanto de la influencia de los centros escolares sobre la inseguridad y la violencia interna como de la posibilidad de actuar sobre un fenómeno que evidentemente no era solo exógeno (Debarbieux; Blaya). Es durante estos años cuando el equipo que dirige el profesor Debarbieux comienza sus investigaciones nacionales, momento en el que el gobierno francés inauguró planes nacionales de lucha contra la violencia escolar. (Blaya, 2004, p. 296).

A este respecto, la experiencia holandesa desarrolló en sus inicios un enfoque de política de seguridad escolar muy centrado en la conducta de los jóvenes:

[...] en Holanda, en concreto, la campaña Escuelas Seguras cuenta con instrumentos para medir la conducta y guiar la acción correspondiente del profesor. Como parte de la campaña, la seguridad de la escuela y de su entorno se está mejorando mediante el trabajo de comités especiales. (Mooij, 1997, p. 42).

Los enfoques de seguridad escolar corresponden o se ajustan a las características de los diferentes países que deciden hacer algo frente a la violencia escolar; en el caso inglés, la revisión sobre sus políticas permite pensar en la ausencia de una concepción de libertad escolar:

Piénsese en el mensaje que se envía a los estudiantes en cuanto a la concepción escolar de confianza y libertad cuando se instalan detectores de metales, videocámaras, dispositivos de identidad, perros rastreadores de drogas, y guardias para garantizar la libertad. En efecto, Ilevados por la seguridad y el cumplimiento de normas, como Thompkins (en este volumen) señala, las escuelas se están convirtiendo más en prisiones que en lugares de aprendizaje. (Henry, 2000, p. 19) ${ }^{2}$.

A pesar de las diferencias que puedan establecerse entre continentes y países, en el fondo el debate de la seguridad escolar es muy similar; aunque con los agravantes de la periferia en la economía-mundo y las limitantes presupuestales de los

\footnotetext{
2 Traducción libre del autor. "Think of the message that is sent to the students in terms of the conception of school trust and freedom when installing metal detectors, video cameras, devices of identity, sniffer dogs of drugs, and guards to ensure freedom. In fact, taken away by the security and compliance, as Thompkins (in this volume) said, schools are becoming more in prisons than in places of learning".
} 
gobiernos latinoamericanos, el campo escolar resulta en una suerte de imbricación del contexto que lo rodea, más las lógicas institucionales regidas por los planes, programas y proyectos educativos del régimen político y del gobierno de turno.

Al sentimiento de inseguridad y la victimización debe sumársele en muchos casos la relación con las políticas de seguridad nacional y ciudadana que se dirigen a contrarrestar el narcotráfico y la delincuencia común asociada con la estigmatización que se hace de los jóvenes en zonas populares.

En un ambiente donde impera la ley del silencio y del más fuerte sobre todo cuando la escuela no dispone de mecanismos de seguridad, de protección y de solución de conflictos - los testigos y las víctimas no comentan lo que ven, lo que saben, por temor a las represalias o del estigma, lo que fortalece la cultura del miedo. Se revela también la vulnerabilidad de los más débiles, resultante de la intimidación física y verbal, banalizando la violencia y haciendo que los diferentes actores se sientan desprotegidos. Eso implica, asimismo, la sensación de inseguridad, desorden y de impunidad, lo que genera la desorganización del espacio público. (Abramovay, 2005, p. 853).

En el caso de Centroamérica, México cuenta con un acervo importante de políticas y programas en seguridad escolar; al ser un país federalista, cada Estado que lo compone tiene autonomía para diseñar sus propias políticas y legislación al respecto, lo que no indica que exista diversidad de propuestas al respecto; es más, parece que la repetición de postulados o directrices es común, más la influencia del gobierno central. Es probable que en México la política más importante en relación al tema sea el "Programa Escuela Segura" (PES). No siendo propiamente una política sino un programa que dependió de una política nacional de seguridad.

El objetivo del PES es: "contribuir a generar en las escuelas de educación básica condiciones que propicien ambientes de seguridad y sana convivencia, favorables para la mejora de los aprendizajes, así como la práctica de valores cívicos y éticos" (SEP, 2013 , p. 3). Para el logro de este objetivo, el programa otorga apoyos económicos y técnicos a las escuelas primarias y secundarias localizadas en zonas con mayor índice de violencia en el país. (Centro de Estudios Educativos, 2014, p. 7).

Por mandato del presidente Felipe Calderón, el programa comenzó en 2007, en el marco de la estrategia "Limpiemos México. Estrategia Nacional de Seguridad. Programa en Zona de Recuperación", como una prioridad en la lucha contra el crimen organizado. En su fase inicial participó el estado de Jalisco, y hoy se encuentra en su fase de generalización y medición de impacto.

La seguridad escolar en México puede ser asociada a la concepción de seguridad nacional y en su contexto específico a convivencia y ciudadanía. En este sentido, hay una relación directa con las violencias macro y microsociales. La categoría en este caso está pensada como estrategia de abordaje del Estado para resolver en parte los problemas de la macro violencia.

Se identifica, entonces, una contradicción entre lo jurídico y su práctica, pues en el campo institucional escolar este tipo de política puede verse como una carga para directivos y docentes, y no como una solución pedagógica a la violencia escolar.

Revisar mochilas, instalar cámaras, poner vigilancia de algún policía, autoridad escolar o familiar en los horarios de entrada y salida, dar pláticas sobre las drogas y el daño que generan son estrategias que no han dado resultados; por el contrario, han generado una percepción negativa y una sensación de desconfianza, principalmente entre las y los estudiantes (Consejo Ciudadano de Seguridad Pública, Prevención y Readaptación Social del estado de Jalisco, 2010; Zurita, 2011). Aunado a esto, existe poco conocimiento de lo que implica el programa por parte de personal directivo y docente, pues es visto como una carga adicional de trabajo al que tienen que apegarse institucionalmente (SEP/Parametría, 2009). De ahí que resulte de especial importancia no solo brindar a las escuelas un acompañamiento permanente en los procesos de operación de este tipo de iniciativas, sino informar, de manera amplia, a los actores educativos y a la sociedad en general acerca de la naturaleza del mismo. (Centro de Estudios Educativos, 2014, p. 8). 
Además para esta política mexicana de seguridad escolar se plantea lo siguiente:

[...] si bien el PES reconoce en sus objetivos la ligazón entre la democracia y la generación de ambientes escolares seguros, no se refiere en su concepción a la CDN en particular ni al conjunto de instrumentos internacionales y nacionales dirigidos a la defensa y promoción de los Derechos de la Infancia y la Adolescencia. (Zurita, 2010, p. 122).

Es importante mencionar que en la configuración de la seguridad escolar como problema político, la influencia de los medios de comunicación y la forma como el Estado legisla alrededor del tema constituyen cómo la sociedad encara el problema de la violencia escolar mediante la implementación de políticas que operan en intereses diferentes al de garantizar la eficacia del derecho de los jóvenes en el campo educativo.

En México existen 27 leyes sobre violencia y seguridad escolar, las cuales

[...] reflejan visiones singulares de la violencia en las escuelas donde algunas insisten (Veracruz, Yucatán, Nuevo León) en considerarla un problema entre niñas, niños, adolescentes y jóvenes. Haciendo caso omiso de la complejidad que encierra este fenómeno y que trasciende la concepción tradicional de la indisciplina estudiantil o del uso excesivo del poder y la autoridad docente. (Zurita, 2015, p. 88).

El alto número de leyes va de la mano con un incremento de noticias sobre violencia escolar en los medios de comunicación, un tinte sensacionalista sobre ellas y una tendencia a respaldar la mano dura frente a la violencia escolar; esto forma parte del análisis que realiza Úrsula Zurita (2015), en México:

[...] el análisis del papel de los medios de comunicación en torno a la violencia escolar permite adentrarse en su desempeño en el contexto de la formulación de los problemas concebidos públicos, con el fin de que sean incorporados en la agenda de las políticas públicas; también propicia un acercamiento a las distintas formas en que se observa y conceptualiza la violencia en las escuelas mexicanas hoy en día. (p. 84).
En el resto de la región latinoamericana existen también diversas miradas al fenómeno de la seguridad escolar; no solo prevalece el enfoque centrado en la prevención e intervención de todo tipo de violencia, sino que la categoría de seguridad escolar permite una relación amplia hacia el sentido de bienestar social en la escuela. Unicef, por ejemplo, diseñó una herramienta para medir el índice de seguridad escolar, a partir de un proyecto piloto en algunos países de Suramérica.

Esta propuesta tiene dos variables fundamentales: la vulnerabilidad de los centros educativos y la reducción del riesgo de desastres. En esta última se encuentran los de tipo social, donde pueden identificarse tipologías de la violencia escolar, pero el factor que amplía la categoría de seguridad escolar es la estructura de las edificaciones y la prevención de desastres naturales. En el contexto suramericano esto es primordial debido a la informalidad en muchos casos de las edificaciones de las instituciones escolares.

Entre los factores que determinan la vulnerabilidad de los centros educativos encontramos, la falta de conocimiento sobre las amenazas presentes en el territorio donde se ubica el centro educativo; equivocados sistemas constructivos, formas de construcción, ampliaciones inadecuadas; falta de inspección y supervisión técnica en los procesos de atención y el alto nivel de deterioro en que se encuentran algunas edificaciones debido a la falta de mantenimiento preventivo y correctivo, contando además la exposición ante diversos riesgos sociales, como la violencia, trabajo infantil y adolescente, drogadicción y otras adicciones, ludopatía, embarazo adolescente, bullying, desnutrición, entre otros. (Unicef, Proyecto DIPECHO VII Suramérica, 2012, p. 2).

Pero la apuesta fundamental de la seguridad escolar, siguiendo su trayectoria como categoría asociada a la mitigación de la violencia escolar, está orientada a su relación con el enfoque de los derechos humanos, aunque se le contraponga el discurso de los problemas sociales asociados al crimen, la inseguridad urbana causada por los jóvenes y los otros problemas que atañen a la seguridad nacional. 
En Chile también se han identificado dos paradigmas antagónicos frente al discurso jurídico de la violencia escolar desde la perspectiva de la seguridad escolar.

Se indica que en la ley coexisten dos paradigmas antagónicos. Un paradigma nominado de "control y sanción", que se funda en la postura de seguridad nacional y corresponde a una concepción conductista de la educación que postula la eficiencia social y la existencia del comportamiento agresivo. Otro paradigma nominado de la "convivencia escolar democrática", que se funda en la concepción de seguridad humana y corresponde a una concepción crítica de la educación que apunta hacia la reconstrucción social y la noción de convivencia escolar. Se presentan las consecuencias que la aplicación de cada uno de estos paradigmas podría tener para el Comité de Buena Convivencia Escolar, el Reglamento interno y el rol del encargado responsable de la convivencia escolar. (Magendzo, Toledo y García, 2012, 377).

Para este país las interacciones en la escuela se constituyen en una problemática social; para el año 2000 se inicia la política de convivencia escolar, hasta el año 2010 se formula la ley antibullying, un proceso de diez años para legislar contra la violencia escolar. Siguiendo los dos paradigmas expuesto en la Ley, se pasa de la perspectiva de la seguridad nacional a la de la seguridad humana. Las diferencias radican en el enfoque centrado en derechos humanos y factores de riesgo que generan inseguridad. Una diferencia entre seguridad ciudadana y seguridad humana, radica en el centramiento operativo que puede tener la primera en las ciudades y lo urbano, mientras la segunda es independiente de la configuración del espacio territorial, solo responde por la vida y la dignidad humana.

Entre 2009 y 2010, en Costa Rica se inició un proyecto denominado Escuela Segura-Comunidad Segura, que incluyó experiencias en cuatro sectores de ciudades de cuatro países: Bolivia, Uruguay, Paraguay y Colombia. Allí se evidenció cómo se logra integrar la categoría de seguridad humana al campo educativo, la escuela y su entorno.
Escuela Segura-Comunidad Segura se basa en el concepto fundamental de seguridad humana que enfatiza el individuo como foco de atención de la comunidad internacional, del Estado y del propio gobierno local. La seguridad humana promueve la protección de los derechos humanos, el predominio de la ley y la democracia, y la satisfacción de necesidades básicas. A la vez, tiene como enfoque fundamental el desarrollo de un proceso de prevención desde el gobierno local, lo cual abarca toda la amplitud territorial del municipio y se observa desde la perspectiva de la seguridad humana, que supera ampliamente el concepto tradicional de seguridad y gestión policial. (BID, Open Society Foundations, Universidad de Chile, 2010, p. 8).

Para Colombia, las leyes de 2006 (Ley 1098, que expide el Código de Infancia y Adolescencia) y 2013 son referentes obligados en la materia de protección de los derechos de los niños y niñas, particularmente la Ley 1620 de 2013 (que formula el Sistema Nacional de Convivencia Escolar y Formación para el Ejercicio de los Derechos Humanos, la Educación para la Sexualidad y la Prevención y Mitigación de la Violencia Escolar). Constituyen una herramienta política clave para el país.

En Bogotá se logra identificar con mayor prontitud directrices sobre seguridad escolar, adelantándose incluso al ámbito nacional. Es probable que esto se deba al abordaje de la seguridad ciudadana en la ciudad, desde los planteamientos de gobiernos distritales que fueron pioneros en Colombia, como el caso del exalcalde Antanas Mockus y su Plan de Desarrollo, "Formar Ciudad" (1995-1998). Allí hay un giro de la política local de seguridad ciudadana, pues cambia la perspectiva influenciada por la violencia política, a una mirada sociocultural de patrones autorregulados por la sociedad; en este caso, la comprendida por la ciudadanía bogotana.

Desde entonces, la seguridad ciudadana será un tema relevante en todos los planes de desarrollo de la ciudad, siendo significativo posteriormente su configuración para el campo educativo a partir de la creación del Sistema Distrital de Seguridad Escolar (Sisdise) a través del Acuerdo 173 de 2005 del Concejo de Bogotá, lo que constituye una directriz relevante para la ciudad en al abordaje de 
la seguridad escolar, actualizándola frente a los avances resolutivos de la temática en el contexto internacional.

\section{La seguridad escolar como problema político en Bogotá}

En el caso colombiano, al igual que en el resto del mundo, la seguridad escolar al presentarse como respuesta al problema social de la violencia escolar se constituye como un problema político y se inscribe en la agenda pública, donde finalmente la controversia y las posturas de actores sociales y políticos evidencian la relación global/sectorial, desde donde se formulan directrices de política pública para la seguridad escolar en Bogotá.

El estudio de la implementación de la política de seguridad escolar brinda grandes posibilidades de abordaje para entender la trayectoria de esta política, sus configuraciones, sus discursos y su impacto frente al problema de la inseguridad escolar. Al igual que en México en la investigación sobre la violencia escolar, "el análisis de la legislación desde una perspectiva de la sociología de la educación y del enfoque de las políticas públicas es todavía incipiente" (Zurita, 2012, p. 22).

Se resalta entonces como interés investigativo para Bogotá optar por el enfoque de análisis de políticas públicas que incluye de hecho el análisis legislativo, aunque interesa en particular el análisis de su implementación, y en el caso colombiano no existe aún un estudio de la seguridad escolar desde esta mirada.

Según Eljach (2011), es necesario incentivar la investigación para comprender la violencia escolar, pero también para que sirva de fundamento para la política pública.

Permitir la utilización de estas investigaciones y estudios para formular políticas públicas y construir indicadores asentados en la realidad; es decir, investigar para promocionar el cambio y poder responder de forma inmediata, pero al mismo tiempo estructural, a la violencia al interior de las escuelas. (p. 114).

El discurso que ha optado la nación colombiana a partir de la Ley 1620 de 2013 se ajusta al enfoque de los derechos humanos. Según Jiménez
(2007), este enfoque aporta a la superación de la visión de derechos por generaciones, por concebirlos como integrales, interdependientes y complementarios, al igual que la concepción de que los derechos se pueden materializar por medio de políticas públicas.

El enfoque de los derechos humanos se concibe como una nueva perspectiva sobre la manera de lograr la materialización de los derechos y elevar la dignidad humana; esto explica su cercanía con los procesos de desarrollo socioeconómico y humano. La perspectiva de derechos también se puede entender como el paso siguiente a las luchas por el reconocimiento positivo-legal de los derechos humanos: si ya están en la constitución, si ya están en los tratados internacionales, es hora de concretarlos. (p. 44).

En consecuencia, la temática de la seguridad escolar se complejiza, pues al asociarse con el enfoque de los derechos toma un carácter transversal que cruza las esferas del bienestar social, esto implicaría entender la seguridad escolar como una expresión del Estado social de derecho, en el sentido de la eficacia del derecho, rebasando el solo contrarrestar o mitigar la violencia escolar.

En la actualidad existen para la ciudad de Bogotá diferentes instrumentos jurídicos que dan cuenta de un abordaje público para la temática de seguridad escolar desde diferentes enfoques o abordajes, pero principalmente responden o buscan intervenir el problema social de la violencia escolar desde la óptica de los derechos humanos.

Diez años han transcurrido desde la creación del Sisdise, y Bogotá cuenta hoy con un "programa de Respuesta integral de orientación escolar" (RIO), que forma parte de un "Proyecto de educación para la ciudadanía y la convivencia" (PECC). Lo anterior tiene como marco dos instancias: la primera corresponde a la política de infancia y adolescencia en Bogotá 2011-2021, que tiene como fundamento el enfoque de derechos; la segunda instancia refiere al Plan de Desarrollo 2012-2016 "Bogotá Humana", cuyo énfasis, el desarrollo humano, da prioridad a la primera infancia con un enfoque diferencial, según lo plantea parte de su objetivo general promocionando las políticas de defensa y protección 
de los derechos humanos. Por último, el "Proyecto de educación para la ciudadanía y la convivencia" es la expresión de la administración anterior por la búsqueda de la garantía al derecho de una educación de calidad.

En este sentido, la Secretaría Distrital de Educación (SED) le ha apostado a la articulación coordinada y eficaz de varios instrumentos que permitan materializar varios acuerdos del Concejo de Bogotá adoptados durante los últimos años en materia de convivencia, seguridad y orientación escolar. Acuerdos que constituyen la expresión política de distintos sectores que han trabajado para el mejoramiento de la educación de las niñas, niños y jóvenes del Distrito Capital. (SED, 2013, p. 4).

Hoy, el discurso de la política se dirige hacia la lógica de la orientación escolar. La estrategia "Respuesta integral de orientación escolar" (RIO) es la expresión de unificar o relacionar las diferentes directrices del Distrito Capital; ella plantea:

[La] normatividad no solo es de especial importancia para la ciudad, sino que constituye un sustento legal, que abarca una serie de insumos pertinentes para acompañar eventos asociados a la convivencia y la ciudadanía que puedan presentarse en los centros educativos, desde una perspectiva amplia de orientación escolar. (SED, 2013, p. 4).

Se busca que esta estrategia responda a las necesidades de garantía, prevención, promoción, difusión y restablecimiento de derechos y al desarrollo de capacidades ciudadanas. La figura 1 muestra los acuerdos que se tuvieron en cuenta.

El avance de la política de seguridad escolar desde la perspectiva de la administración distrital Bogotá Humana es relevante, pues el discurso se ha fortalecido en el enfoque de los derechos humanos,

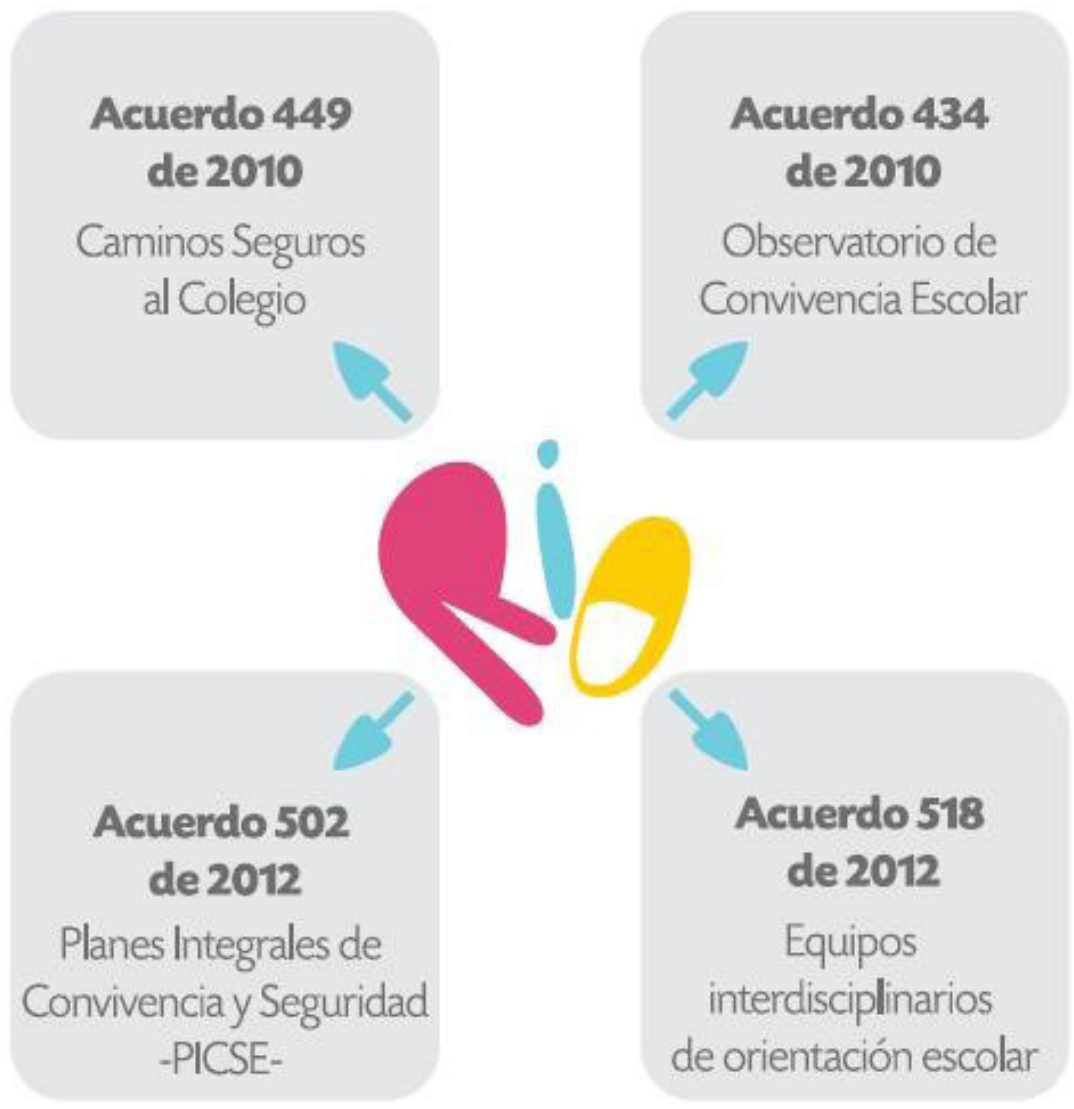

Figura 1. Acuerdos distritales.

Fuente: SED (2013, p. 5). 
ha sido un trasegar importante en la medida que se articulan discursos como la convivencia escolar, entornos seguros y orientación escolar.

La Secretaría de Gobierno de Bogotá realizó un estudio en 2007 sobre la situación de la convivencia y seguridad con más de 800.000 estudiantes, los datos del informe son presentados en la siguiente tabla, siendo relevantes en porcentaje estadístico el hurto y el maltrato emocional.

El presente informe contiene el análisis general de las estadísticas descriptivas que resultaron de esta encuesta luego de su expansión por el DANE a la población escolar total matriculada en los grados considerados por el estudio (826.455 estudiantes) en los planteles escolares de Bogotá y municipios aledaños. (Secretaría de Gobierno, 2007).

Sin embargo, a pesar de la claridad de las directrices políticas, la realidad de los problemas de violencia escolar e inseguridad siguen siendo relevantes y no hay una clara disminución del fenómeno. Para 2013 la SED realizó la Encuesta de clima escolar y victimización, en ella

[...] se encuestaron 118.000 estudiantes en total, que representaron a las y los 568.000 estudiantes, total de estudiantes de los colegios públicos y privados en esos grados. Del total de encuestados 86.500 son de colegios públicos y representaron a 377.000 estudiantes, y 31.400 de colegios privados, que representaron a 191.000 estudiantes. (p. 15).

La encuesta es un buen referente de la situación actual del campo educativo frente al tema de la seguridad escolar, pues los planes, programas y proyectos al respecto tienen un alto grado de integralidad y responden de forma amplia a las necesidades y requerimientos de la población escolar y sus entornos; sin embargo, la situación desde 2006 tiende a empeorar en algunos aspectos como el entorno, y en otros se mantiene. La Secretaría de Gobierno realizó la encuesta ese año con factores similares, incluso la encuesta de 2011 realizada por la SED se ajusta a esta tendencia. En el $52 \%$ de los colegios públicos, el $20 \%$ o más de los encuestados manifestaron que al menos una vez los habían atracado. Este reporte solo se da en el $22 \%$ de los colegios privados (SED; Secretaria Distrital de Cultura, Recreación y Deporte, y Universidad Distrital Francisco José de Caldas, 2013, p. 72).

El marco legislativo en el tema para Colombia y la capital, las acciones distritales frente al tema en los últimos tres gobiernos en el marco de diez años desde el Acuerdo 173 de 2005, las mediciones e indicadores de las diferentes encuestas en la

Tabla 1. Conclusiones y recomendaciones.

\begin{tabular}{|c|c|c|}
\hline TIPO DE VIOLENCIA & PRESENCIA & SUGERENCIAS \\
\hline $\begin{array}{l}\text { Hurto menor sin violencia en contexto } \\
\text { escolar }\end{array}$ & $56 \%$ & $\begin{array}{l}\text { Investigaciones cualitativas en } \\
\text { contextos específicos }\end{array}$ \\
\hline Maltrato emocional: insultos & $38 \%$ & \multirow{2}{*}{$\begin{array}{l}\text { Intervenciones orientadas a mejorar el } \\
\text { respeto y la confianza }\end{array}$} \\
\hline Maltrato emocional: exclusión por pares & $22 \%$ & \\
\hline Bullying & $\begin{array}{l}15 \% \text { compañero de curso, } 12 \% \\
\text { otro curso }\end{array}$ & Hacerla más visible \\
\hline Acoso sexual verbal & $\begin{array}{l}13 \% \text { compañero de curso, } 9 \% \\
\text { otro curso }\end{array}$ & \\
\hline Contactos sexuales no deseados & $10 \%$ & \\
\hline Armas blancas & $9 \%$ & Desincentivar porte de armas \\
\hline Percepción dentro y fuera de la escuela & $11 \%$ dentro, $28 \%$ fuera & Protección de la ruta de los escolares \\
\hline Consumo de sustancias ilícitas & $33 \%$ alcohol, $3 \%$ drogas & Procesos de comunicación pública \\
\hline
\end{tabular}

Fuente: elaboración propia a partir de Secretaría de Gobierno (2007, pp. 5-7). 
ciudad, el impacto mediático de los últimos años sobre la violencia escolar, los estudios y enfoques de análisis del problema desde la investigación sobre educación, permiten entender a la seguridad escolar como un problema político.

\section{Conclusiones}

La seguridad escolar es relevante por cuanto se ubica en el campo educativo como una condición esencial para su funcionamiento, involucra todos los agentes sociales del campo, trasciende la comprensión endógena del mismo y se orienta en un discurso universal que es aprehendido por todas las agencias internacionales en educación y asumido por todos los Estados de derecho en el mundo. Los derechos humanos y su expresión centrada en la condición de sujetos de derecho de los niños, niñas y adolescentes, constituye ese discurso orientador. Esta apuesta avanza y se sobrepone al enfoque centrado en la coerción y en la sola expresión del control social ejercido en la escuela sobre la infancia,

22 los jóvenes y los maestros, en esencia, todos los agentes contenidos en el campo.

Una escuela insegura hace que la promesa del desarrollo social y económico de una sociedad sea un proyecto cada vez más difícil de cumplir, pues aun teniendo escuelas seguras, con un clima social favorable para los procesos de aprendizaje y escolarización no son garantía de éxito, por cuanto el progreso de una sociedad no recae tan solo en lo que la institución escolar pueda hacer en el pensamiento y la cultura de niños, niñas y jóvenes, pues el mundo de los adultos se antepone en muchos casos a su nueva condición de sujetos de derechos, instaurada en la convención de los derechos de los niños en 1989.

La seguridad escolar puede pensarse incluso como garantía social para el funcionamiento de un sistema escolar, inclusive es también un dispositivo de control social para niños, niñas y adolescentes en aras de mitigar la violencia escolar y otros factores que amenazan el aprendizaje, durante el tiempo de su escolarización. En esencia, la seguridad escolar cobra valor pedagógico al asumir el enfoque de derechos humanos y ser garante de su condición de sujetos de derecho, lo anterior implica el uso de la autonomía relativa a todo campo social, para apartarse de privilegiar en sus prácticas sociales la lógica de protección al mercado y su producción de sujetos de consumo.

Entender la seguridad escolar como un problema político requiere la atención de todos los actores de la sociedad involucrados directa o indirectamente con el campo educativo y la escuela, en particular de los Estados como responsables directos de la materialización de los derechos humanos en sus países, responsabilidad que se implementa a través de sus legislaciones y políticas públicas. Estas últimas manifiestan el estado actual de las intervenciones públicas para brindar seguridad escolar, y en últimas son una imagen de la comprensión de una sociedad particular sobre el capital simbólico que le atribuyan a su institucionalidad educativa.

Los estudios sobre implementación de políticas reconocen la necesidad de profundizar en el análisis del ciclo de las políticas, pues cada fase de este está interconectada con la siguiente, lo cual complejiza el objeto de la política pública, es decir, el problema político. En este caso, la seguridad escolar — que termina siendo una respuesta sociopolítica que combina el esfuerzo por luchar contra la violencia escolar y garantizar la aplicación de los derechos humanos y la condición de sujetos de derecho de los niños - implica entender su lógica de construcción, como un asunto relevante de carácter político y educativo.

El análisis de la política de seguridad escolar, después de una década de implementación en Bogotá, es una tarea investigativa que puede aportar a la reformulación de la política, a encontrar correspondencias y vacíos con el enfoque de derechos en el campo educativo, permitir una mirada crítica de las acciones coercitivas sustentadas en la doctrina de seguridad nacional en la escuela, dar cuenta de las prácticas sociales asociadas a la seguridad escolar desde los propios agentes del campo escolar.

La seguridad escolar puede ser identificada como un estado de confianza, un valor social que se mueve en las lógicas de la percepción, en las experiencias vividas de los agentes escolares, incluso puede ser asociada a prácticas económicas proclives a la rentabilidad del sistema escolar. Garantizar el estado de confianza en las instituciones escolares, a partir de las diversas prácticas sociales que allí se generan apuntaría a la búsqueda de la 
calidad en la educación. Sin embargo, aún el dilema está en ajustarse al discurso jurídico como garantía del respeto a los derechos humanos, cuando contrario al mismo se aplican medidas de seguridad en un enfoque coercitivo, vigilantista y punitivo, cercano a la doctrina de seguridad nacional trasgrediendo el sentido pedagógico de toda acción de bienestar en el campo educativo.

Queda manifiesto entonces que en los últimos años se ha pensado el problema de la violencia escolar desde la acción pública y que la configuración de dicha respuesta estatal se mueve desde la perspectiva de la seguridad escolar, la convivencia, y más reciente de la orientación escolar, todas articuladas por el enfoque de derechos de los niños, pero también influenciada por la doctrina de la seguridad nacional que se equipara a la seguridad ciudadana, como respuesta a los problemas subyacentes de violencia social relacionados con el campo escolar, presentando una controversia de enfoque e implementación con las apuestas del paradigma de la seguridad humana.

\section{Referencias}

Abramovay, M. (2005). Victimización en las escuelas. Ambiente escolar, robos y agresiones físicas. Revista Mexicana de Investigación Educativa, 10(26): 833-864.

Angarita, P. (2002). La seguridad ciudadana: nuevo reto en la defensa de los derechos humanos. En: Derechos Humanos, seguridad ciudadana y sociedad civil. Sistematización de experiencias (pp. 117-166). San José, Costa Rica: IIDH, Instituto Interamericano de Derechos Humanos.

Banco Interamericano de Desarrollo (BID), Open Society Foundations, Universidad de Chile. (2010). Banco de buenas prácticas en prevención del delito en América Latina y el Caribe. San José, Costa Rica: Fundación Arias para la Paz y el Progreso Humano.

Blaya, C. (2004). Clima y violencia en la escuela. Un estudio comparativo entre España y Francia. Revista de Educación, 339: 293-315.

Blaya, C. (2012). Violencia escolar. ¿Una juventud desilusionada? En: A. Furlan. Reflexiones sobre la violencia en las escuelas (pp. 31-44). México: Siglo XXI.
Borda, E.; Rodríguez, C.; Libreros, J. (2004). Conflicto y seguridad democrática en Colombia. Temas críticos y propuestas. Bogotá: Fescol, Fundación Social.

Camacho, G. A. (1994). Seguridad. ¿Para la gente o para el Estado? Análisis Político, 21: 80-95.

Centro de Estudios Educativos, A.C. (abril-junio de 2014). Más allá de Escuela Segura. Revista Latinoamericana de Estudios Educativos, xLIV(2): 5-12.

Concejo de Bogotá. (2005). Acuerdo 173 de 2005. Sistema Distrital de Seguridad Escolar "SISDISE". Bogotá, Colombia.

Debarbieux, E. (mayo-agosto de 1997). La violencia en la escuela francesa: análisis de la situación, políticas públicas e investigaciones. Revista de Educación, 313: 79-94.

Delgado, J. (1997). El tránsito hacia un nuevo sistema de seguridad. En: G. Segobia. Justicia, seguridad y convivencia en Santa Fe de Bogotá. Bogotá: Consejería Presidencial para el Desarrollo Territorial.

Eljach, S. (2011). Violencia escolar en América Latina y el caribe. Superficie y fondo. Panamá: Unicef y Plan.

Fondo de las Naciones Unidas para la Infancia (Unicef). (julio de 2005). Convención sobre los Derechos del Niño. Bogotá, Colombia.

Fondo de las Naciones Unidas para la Infancia (Unicef), Proyecto DIPECHO VII Suramérica. (2012). Índice de seguridad escolar. Guía de implementación. Panamá: Unicef.

Fuentes, C.; Rojas, F. (2005). Promover la seguridad humana: marcos éticos, normativos y educacionales en América Latina y el Caribe. París: Unesco.

Gvirtz, S.; Larrondo, M. (2012). Democracia, diálogo. Construcción de la ley. Caminos para construir la convivencia en el espacio escolar y generar modos no violentos de relación. En: A. Furlán. Reflexiones sobre la violencia en las escuelas (pp. 295-312). México: Siglo XXI.

Henry, S. (2000). What is school violence? An integrated definition. Annals of the American Academy of Political and Social Science, 567: 16-29. 
Jiménez, A. (enero-junio de 2013). El problema de la institucionalización del Estado en Michel Foucault. Esfera, 3(1): 14-27.

Jiménez, W. (2007). El enfoque de los Derechos Humanos y las políticas públicas. Civilizar, 7(12): 31-46.

Leal, F. (1994). Seguridad nacional y seguridad ciudadana, violencia urbana e inseguridad ciudadana. Santa Marta, Colombia: PNUD-PNR.

Magendzo, A.; Toledo, M.; García, V. (2012). Descripción y análisis de la Ley sobre violencia escolar (20.536): dos paradigmas antagónicos. Estudios Pedagógicos, XXXIX: 377-391.

Monclús, A.; Saban, C. (2006). Violencia escolar: actuaciones y propuesta a nivel internacional. Barcelona: Davinci Continental.

Mooij, T. (1997). Por la seguridad en la escuela. Revista de Educación, 313: 29-52.

Organización de Estados Americanos (OEA). (2003). Declaración sobre seguridad en las Américas. Conferencia especial sobre seguridad (pp. 1-14). México.

Pérez, K. (2007). El concepto y el uso de la seguridad humana: análisis crítico de sus potencialidades y riesgos. CIDOB d'Afers Internacionals, 76: 59-77.

Ruiz, L. D.; Amariles, C. (2006). La escuela: territorio en la frontera. Tipología de conflictos escolares según estudio comparado en Bogotá, Cali y Medellín. Medellín: Instituto Popular de Capacitación, Corporación de Promoción Popular.

Secretaría de Educación del Distrito, Secretaría Distrital de Cultura, Recreación y Deporte, Universidad Distrital Francisco José de Caldas. (2013). Encuesta de clima escolar y victimización. Bogotá.

Secretaría de Educación Distrital (SED). (2013). Respuesta integral de orientación escolar (RIO). Recuperado de: http://www.redacademica. edu.co/archivos/redacademica/proyectos/pecc/ centro documentacion/caja de herramientas/ serie 1 orientaciones/03 que es rio.pdf

Secretaría de Gobierno. (2007). Convivencia y seguridad en ámbitos escolares de Bogotá D.C. Bogotá. Recuperado de: http:// www.elespectador.com/files/d8f4461c07272f8357a73012230992f3.pdf

Zurita, U. (2010). Los desafíos del Programa Escuela Segura en el Vigésimo Aniversario de la Convención sobre los Derechos del Niño. Rayuela. Revista Iberoamericana sobre Infancia y Juventud en Lucha por sus Derechos 1(2), 118-124.

Zurita, U. (2012). Las escuelas mexicanas y la legislación sobre la Convivencia, la Seguridad y la Violencia Escolar. Educación y Territorio 2(1), 19-36.

Zurita, U. (2015). La violencia escolar desde los medios de comunicación y el poder legislativo en México. Revista Sophia, 11(1): 81-93. 